\title{
Transport of Reactive Oxygen and Nitrogen Species across Aquaporin: A Molecular Level Picture
}

\author{
Maksudbek Yusupov (D), ${ }^{1}$ Jamoliddin Razzokov, ${ }^{1}$ Rodrigo M. Cordeiro, ${ }^{2}$ \\ and Annemie Bogaerts ${ }^{1}$ \\ ${ }^{1}$ Research Group PLASMANT, Department of Chemistry, University of Antwerp, Universiteitsplein 1, B-2610 Antwerp, Belgium \\ ${ }^{2}$ Centro de Ciências Naturais e Humanas, Universidade Federal do ABC, Avenida dos Estados 5001 CEP 09210-580 Santo André, \\ SP, Brazil
}

Correspondence should be addressed to Maksudbek Yusupov; maksudbek.yusupov@uantwerpen.be

Received 20 April 2019; Accepted 22 May 2019; Published 17 June 2019

Academic Editor: Angel Catalá

Copyright (C) 2019 Maksudbek Yusupov et al. This is an open access article distributed under the Creative Commons Attribution License, which permits unrestricted use, distribution, and reproduction in any medium, provided the original work is properly cited.

\begin{abstract}
Aquaporins (AQPs) are transmembrane proteins that conduct not only water molecules across the cell membrane but also other solutes, such as reactive oxygen and nitrogen species (RONS), produced (among others) by cold atmospheric plasma (CAP). These RONS may induce oxidative stress in the cell interior, which plays a role in cancer treatment. The underlying mechanisms of the transport of RONS across AQPs, however, still remain obscure. We apply molecular dynamics simulations to investigate the permeation of both hydrophilic $\left(\mathrm{H}_{2} \mathrm{O}_{2}\right.$ and $\left.\mathrm{OH}\right)$ and hydrophobic $\left(\mathrm{NO}_{2}\right.$ and $\left.\mathrm{NO}\right)$ RONS through AQP1. Our simulations show that these RONS can all penetrate across the pores of AQP1. The permeation free energy barrier of OH and $\mathrm{NO}$ is lower than that of $\mathrm{H}_{2} \mathrm{O}_{2}$ and $\mathrm{NO}_{2}$, indicating that these radicals may have easier access to the pore interior and interact with the amino acid residues of AQP1. We also study the effect of RONS-induced oxidation of both the phospholipids and AQP1 (i.e., sulfenylation of $\mathrm{Cys}_{191}$ ) on the transport of the above-mentioned RONS across AQP1. Both lipid and protein oxidation seem to slightly increase the free energy barrier for $\mathrm{H}_{2} \mathrm{O}_{2}$ and $\mathrm{NO}_{2}$ permeation, while for $\mathrm{OH}$ and $\mathrm{NO}$, we do not observe a strong effect of oxidation. The simulation results help to gain insight in the underlying mechanisms of the noticeable rise of CAP-induced RONS in cancer cells, thereby improving our understanding on the role of AQPs in the selective anticancer capacity of CAP.
\end{abstract}

\section{Introduction}

In recent years, cold atmospheric plasma (CAP) application in cancer treatment has shown beneficial effects [1]. Experiments already evidenced that CAP may selectively eliminate cancer cells, leaving homologous normal cells less damaged [2-5]. This and other features of CAP, such as causing no pain in patients, no thermal and electrical damage, and low cost $[6,7]$, might give an advantage to CAP over traditional anticancer therapies.

CAP generates reactive oxygen and nitrogen species (ROS and RNS or RONS), e.g., $\mathrm{H}_{2} \mathrm{O}_{2}, \mathrm{OH}, \mathrm{NO}, \mathrm{NO}_{2}$, and $\mathrm{O}_{3}$. It is generally believed that the selective anticancer capacity of CAP is linked to the higher levels of RONS that are generated in cancer cells, while normal cells experience a relatively modest increase (if any) in RONS levels [4, 8-10]. The CAP-induced RONS diffuse across the cell membrane, causing nitrooxidative stress in the cell, thereby affecting the intracellular signaling pathways, and eventually leading to cell death $[4,11,12]$. However, the underlying mechanisms of the distinct increase of intracellular RONS are still not fully understood, although several explanations have already been proposed in the literatures [13-15].

One of the theories to explain the selective rise of intracellular RONS in cancer cells is based on the aquaporin (AQP) transmembrane proteins [13]. AQPs are mainly responsible for transporting water molecules across the membrane, but they can also conduct other permeants (such as $\mathrm{H}_{2} \mathrm{O}_{2}, \mathrm{CO}_{2}$, and $\mathrm{NO}$ ) through their channels $[16,17]$. AQPs are overexpressed in many cancer cells, including 
glioma, hemangioblastoma, lung adenocarcinoma, and laryngeal, colorectal, and ovarian cancer [18]. Recent experiments using AQP8 gene silencing in glioblastoma cells resulted in reduced toxicity of CAP-treated media towards these cancer cells [19]. Moreover, Miller et al. [20] found that the expression of AQP3 on colon adenocarcinoma HT29 cells, mammalian HEK 293 cells, and cervical cancer HeLa cells significantly enhanced the transport of $\mathrm{H}_{2} \mathrm{O}_{2}$ in these cells. Based on these observations, Yan et al. proposed that CAP-induced RONS may permeate into cancer cells considerably faster than into normal cells, through AQPs [13]. Hence, this difference in transmembrane permeation may lead to higher intracellular RONS concentrations, thereby resulting in cancer cell death $[13,19]$.

Following these considerations, we investigate here the transport of RONS across the AQP transmembrane protein, by means of molecular dynamics (MD) simulations. In addition, we consider the effect of CAP-induced oxidation on the transport of these RONS. Some simulation studies have already been performed, which are aimed at understanding the molecular level mechanisms of water conduction by AQPs (see, e.g., [21-25]). Besides, several studies were devoted to the permeation of different nonaqueous solutes across AQPs [26-30]. Cordeiro studied the permeation of ROS (specifically $\mathrm{H}_{2} \mathrm{O}_{2}, \mathrm{HO}_{2}$, and $\mathrm{OH}$ ) across both mammalian and plant AQP models [31]. He found that all these species can permeate across the AQPs, with lower free energy barriers compared to those across the phospholipid bilayer (PLB) [31]. We recently investigated the transport of $\mathrm{H}_{2} \mathrm{O}_{2}$ across AQP1 [32]. Our simulations revealed that the permeability of $\mathrm{H}_{2} \mathrm{O}_{2}$ across AQP1 is at least two orders of magnitude higher than across the PLB, indicating that the delivery of $\mathrm{H}_{2} \mathrm{O}_{2}$ into the cell interior should be across AQP [32]. However, the overall mechanism of RONS permeation across AQP is still obscure. Moreover, there has been no systematic study on the effect of oxidation of both the PLB and AQP on the transport of RONS through AQP. This is of course highly relevant for a better understanding of the selective anticancer capacity of CAP, which produces various RONS and thus induces oxidation, as well as for other cancer therapies that are based on oxidation, such as chemotherapy, radiotherapy, and photodynamic therapy.

As the CAP-generated RONS reach the cell membrane, some are expected to enter the intracellular medium via AQPs or simple transmembrane diffusion. However, both the PLB and the AQPs are themselves targets to RONSinduced oxidation. It is conceivable that oxidation may change the properties of the PLB and the AQPs, which in turn generates a feedback loop on RONS permeability. In fact, experiments have shown that the AQP conductivity can be reversibly inhibited upon exposure to ROS [33-35]. It was proposed that direct oxidation of AQP or its surrounding phospholipids could trigger conformational changes that lead to channel closure. Recovery of the conductive state would then depend on cellular repair mechanisms. However, the existence of an oxidative gating mechanism remains to be demonstrated at the molecular level. It is also conceivable that, depending on the nature and extent of the oxidation process, the AQP permeability may be enhanced.
In this study, we carry out MD simulations to investigate the transport of RONS (specifically $\mathrm{H}_{2} \mathrm{O}_{2}, \mathrm{OH}$, $\mathrm{NO}_{2}$, and $\mathrm{NO}$ ) across the transmembrane protein AQP1 with molecular detail, as well as the effect of oxidation of both the PLB and AQP1 themselves. Specifically, we calculate the free energy profiles (FEPs) of RONS across (i) native AQP1, (ii) AQP1 surrounded by $50 \%$ oxidized phospholipids, and (iii) AQP1 containing oxidized Cys residues (see below).

\section{Computational Details}

We performed MD simulations of the permeation of $\mathrm{H}_{2} \mathrm{O}_{2}$, $\mathrm{OH}, \mathrm{NO}_{2}$, and $\mathrm{NO}$ across AQP1. AQP1 is one of the members of the AQP family and widely expressed in different cancerous tissues, including breast cancer, colorectal cancer, astrocytoma, ovarian cancer, cervical cancer, and lung cancer [13]. We selected the above-mentioned species, to include two hydrophilic $\left(\mathrm{H}_{2} \mathrm{O}_{2}\right.$ and $\left.\mathrm{OH}\right)$ and two hydrophobic $\left(\mathrm{NO}_{2}\right.$ and $\left.\mathrm{NO}\right)$ representative RONS that are important in CAP treatment of cancer $[11,36]$.

Furthermore, we considered the AQP1 model in three possible states, called NAT, OXL, and OXP. NAT stands for native AQP1 (pdb id: 1J4N [37]) embedded in a fully hydrated native palmitoyl-oleoyl-phosphatidylcholine (POPC) bilayer (see Figures 1(a), 1(b), and 1(d)). We chose POPC as a model PLB because it is in the fluid state at the temperature applied in our simulations [38]. OXL also represents the native AQP1, but surrounded by an equimolar mixture of native and oxidized phospholipids. The oxidized phospholipid was considered as the product of oxidative acyl chain cleavage of POPC, which leads to lipid fragments bearing the aldehyde functional group (ALD) (see Figure 1(d)). This is indeed one of the major oxidation products [39]. It is formed from a ring closure and opening reaction of the intermediate lipid peroxide radical, which results in two aldehydes. Finally, OXP consists of oxidized AQP1 (obtained by modification of $\mathrm{Cys}_{191}$ of each monomer to Cys sulfenic acid, i.e., Cys-SOH, see Figures 1(a) and 1(c)), surrounded with native POPC PLB. Thus, NAT denotes the model system containing native AQP and PLB, whereas OXL and OXP represent the systems with either oxidized lipids or oxidized protein, respectively.

As is clear from Figure 1, AQP1 consists of four monomers each acting as an individual channel through which the transport of water and other solutes (e.g., $\mathrm{H}_{2} \mathrm{O}_{2}$ and NO) takes place. These monomers interact with each other through van der Waals forces and hence form the tetrameric complex. At the center of each monomer channel, two highly conserved Asn-Pro-Ala (NPA) motifs are located, which provide the selectivity against the permeation of $\mathrm{H}^{+}$ and other ions [31, 41]. Moreover, near the extracellular part of each channel, there is a constriction region, the so-called aromatic/Arg ( $\mathrm{ar} / \mathrm{R})$, which also contributes to the selectivity. Note that in our simulations the position $z=0$ of each pore channel was set at the NPA region (see NPA and ar/R regions in Figure 2). More information about the structure of AQP1 can be found in $[21,37,42,43]$. 


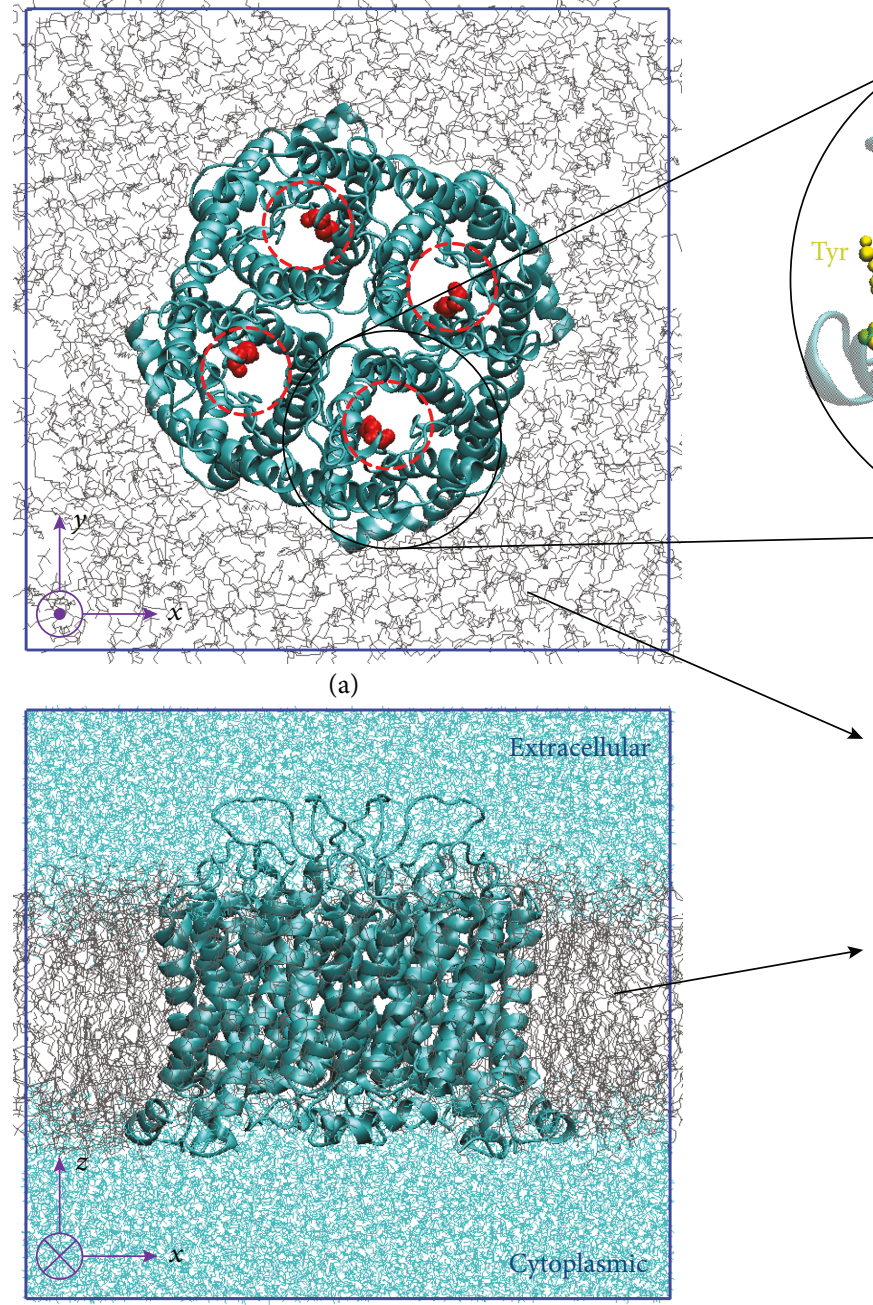

(b)<smiles>NC(CS)C(=O)O</smiles>

Cys<smiles>NC(CSO)C(=O)O</smiles>

Cys-SOH

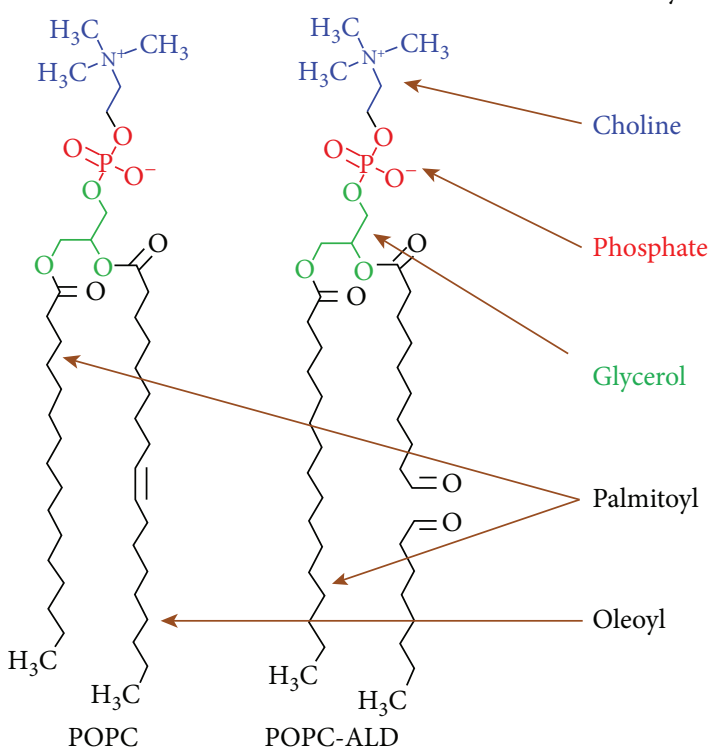

(d)

FIgUre 1: (a) Top and (b) side view of the NAT model system, i.e., the native AQP1 tetramer surrounded by a native POPC PLB (gray color) and covered with water layers on top and at the bottom (cyan color in (b)). The top and bottom water layers correspond to the extracellular and cytoplasmic aqueous regions. For clarity, the water layers are removed in (a). (c) Highlight of some of the AQP amino acid residues that are most reactive with $\mathrm{OH}$ radicals [40]. Cys ${ }_{191}$ is indicated with red dashed circles in (a) and (c). The chemical structures of Cys and its oxidized form Cys sulfenic acid (i.e., Cys-SOH) are given in (c), whereas the chemical structures of POPC and its oxidized form POPCALD are shown in (d). These lipids are used to construct the OXL model system, whereas Cys-SOH residues are used to build the OXP model system. Note that $\mathrm{Cys}_{191}$ is selected as the oxidation target in OXP because it can easily be oxidized and stays close to the ar/R selectivity region (see Figure 2).

\subsection{Preparation of the Model Systems. To create the NAT} model system, we applied the CHARMM-GUI web server $[44,45]$, where the orientation of the AQP1 tetramer into the surrounding PLB was determined by the OPM database [46]. To construct the OXL model system, we used the PACKMOL package [47]. First, AQP and the POPC lipids were packed together and then $50 \%$ of the POPC molecules were replaced by POPC-ALD lipids. Finally, to build the OXP model system, we modified $\mathrm{Cys}_{191}$ in each monomer of the NAT system to Cys-SOH (Figures 1(a) and 1(c)) using the web server Viena-PTM 2.0 [48]. A detailed explanation of the reason for choosing a degree of oxidation of 50\% in OXL and $\mathrm{Cys}_{191}$ as the oxidized amino acid in OXP is given in the Supplementary Information. Briefly, we used 50\% oxidation in OXL, as it is large enough to observe the effect of oxida- tion. Moreover, we selected $\mathrm{Cys}_{191}$ for oxidation in OXP, since it can easily be oxidized and stays close to the ar/R selectivity region.

All model systems were energy minimized using the steepest descent algorithm. Subsequently, they were equilibrated for $150 \mathrm{~ns}$ in the isothermal-isobaric (NPT) ensemble at 310 $\mathrm{K}$ and $1 \mathrm{~atm}$, applying the Nose-Hoover thermostat [49] and the semi-isotropic Parrinello-Rahman barostat [50]. We verified that this equilibration time was sufficient, as the systems reached stability after $\sim 60 \mathrm{~ns}$ (cf. Supplementary Fig. S1a). In all systems, $\mathrm{Cl}^{-}$counter ions were added to the water phase to keep the systems electrically neutral. Periodic boundary conditions were applied in all Cartesian directions. The last $50 \mathrm{~ns}$ of the equilibration time was used to compute the average properties and to extract the starting configurations for 

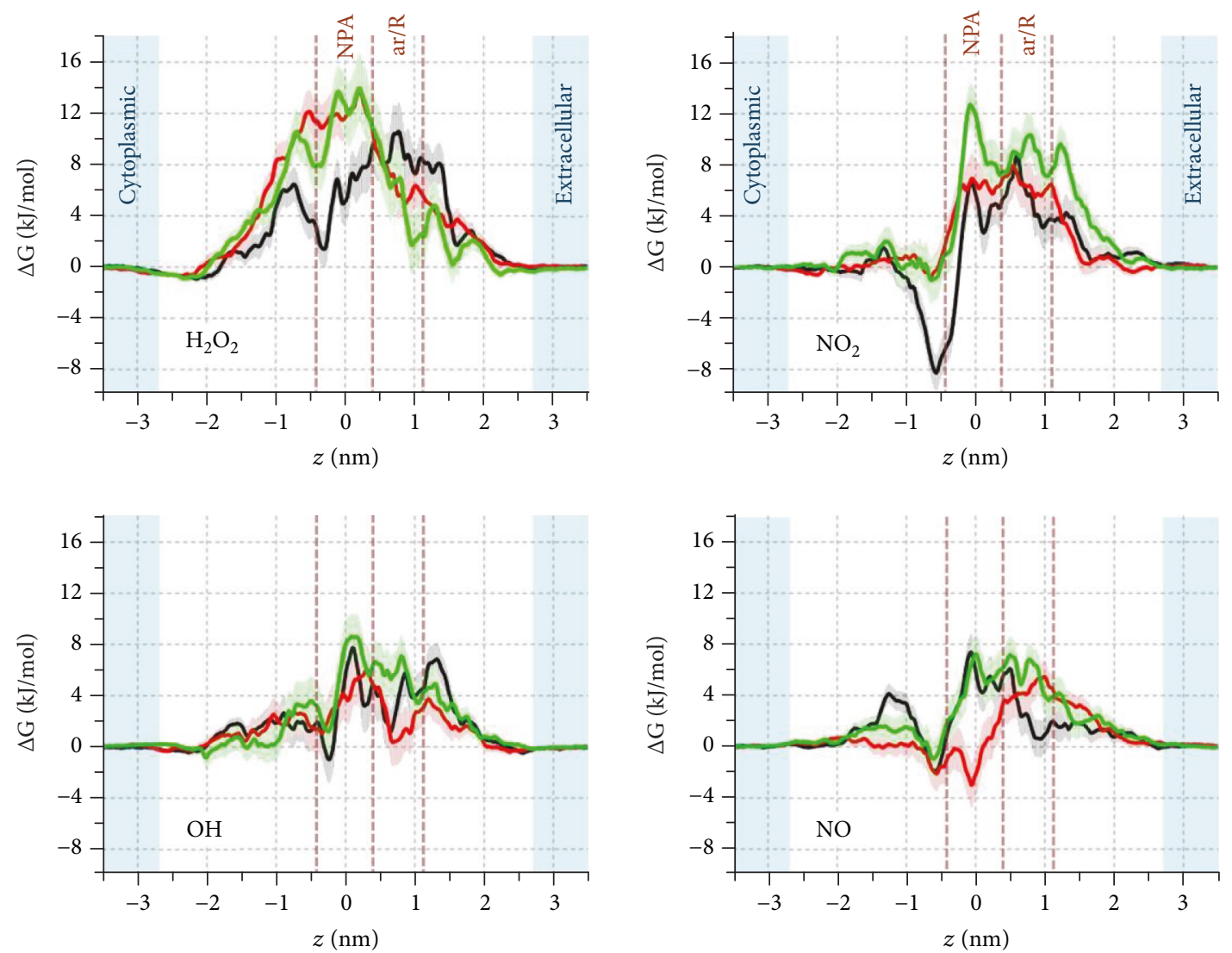

Hydrophilic

Hydrophobic

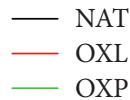

FIgure 2: Average FEPs of the hydrophilic $\left(\mathrm{H}_{2} \mathrm{O}_{2}\right.$ and $\left.\mathrm{OH}\right)$ and hydrophobic $\left(\mathrm{NO}_{2}\right.$ and $\left.\mathrm{NO}\right)$ RONS across AQP1 pores of the native (NAT, black curve) and oxidized (OXL and OXP, red and green curves) model systems. The extracellular and cytoplasmic aqueous phases are shown in light blue color. The uncertainties in the profiles are represented in pale color. The NPA and ar/R regions are indicated by the brown dashed lines, and $z=0 \mathrm{~nm}$ is set to the NPA region. The same designation of the NPA and ar/R regions applies to the other similar figures below.

umbrella sampling (US) simulations (see below). The MD trajectories were recorded at intervals of 20 ps. Both the equilibration and US simulations were carried out with a time step of $2 \mathrm{fs}$.

We used the GROMOS 54A7 force field parameters [51] for the interatomic interactions, in combination with the SPC water model [52]. Moreover, the GROMOS-type parameters for the RONS, the aldehyde products of the oxidized POPC (i.e., POPC-ALD), and the oxidized form of Cys (i.e., Cys-SOH) were obtained from [53-55], [56, 57], respectively. In all simulations, we used a cut-off of 1.1 $\mathrm{nm}$ for both the electrostatic and van der Waals interactions. We selected this cut-off after performing a series of test runs and checking that it led to stable protein structures (cf. convergence of the atomic root mean square displacements in Supplementary Fig. S1a) and a PLB with area per lipid and thickness compatible to reference experimental data [58]. Moreover, using this cut-off, we obtained a very similar FEP of $\mathrm{H}_{2} \mathrm{O}_{2}$ across native AQP1 to our previously obtained FEP [32], which was not the case with other cut-off radii. All simulations and analyses of the results were performed with the GROMACS 5.1.2 package [59], except for the calculation of the pore profiles (see Section 2.2). The illustrations of the simulated systems were prepared with the VMD software [60].

2.2. Calculation of the Pore Profiles. We used the HOLE program [61] to calculate the pore profiles across AQP1. We calculated the average radius of the cavity of each model system along the $z$-direction, i.e., the direction passing through the pore channels (cf. Figures 1(a) and 1(b)). The program proceeds along the planes perpendicular to the direction of the channel vector (i.e., $z$-direction) and finds the largest sphere in each of these planes without overlapping with the van der Waals surface of any atom. Using the AMBER van der Waals atomic radii, we computed the final pore profile of each model system (see Supplementary Fig. S1b) by averaging over 250 individual pore profiles, obtained from the last $50 \mathrm{~ns}$ of the equilibration runs.

2.3. Calculation of the Free Energy Profiles (FEPs). We used the US method [62] to calculate the FEPs of $\mathrm{H}_{2} \mathrm{O}_{2}, \mathrm{OH}$, 
$\mathrm{NO}_{2}$, and $\mathrm{NO}$ across the AQP channels of the three model systems, i.e., NAT, OXL, and OXP. To obtain the average FEP for each permeant and each model system, we used six starting configurations, which were derived from the last 50 ns of equilibration runs (i.e., at $0,10,20,30,40$, and $50 \mathrm{~ns}$ ). In each model system, we defined 72 umbrella windows along the $z$-axis at intervals of $0.1 \mathrm{~nm}$. In this manner, the sampling windows spanned the whole channel ranging from the extracellular to the cytoplasmic aqueous regions (see Figures 1(b) and 2). The solutes were then inserted at the umbrella centers, and the restraining potentials were applied between the center of mass of the solutes and the alpha carbons of the NPA regions. The solutes were restrained to move along the $z$-axis by applying a harmonic bias with a force constant of $2000 \mathrm{~kJ} \cdot \mathrm{mol}^{-1} \cdot \mathrm{nm}^{-2}$. Moreover, their lateral motion in the $x y$-plane was also restrained by using the socalled flat-bottomed position restraint, with a radius of 0.5 $\mathrm{nm}$ and a force constant of $500 \mathrm{~kJ} \cdot \mathrm{mol}^{-1} \cdot \mathrm{nm}^{-2}$. The latter allowed us to insert four solutes in each plane (or umbrella window), each of which corresponding to one pore (cf. Figure 1(a)). During each US simulation, six umbrella windows, separated by $1.2 \mathrm{~nm}$, were sampled simultaneously. Thus, in each US simulation, we were able to obtain the results for 24 solutes (e.g., $24 \mathrm{H}_{2} \mathrm{O}_{2}$ molecules), distributed over six layers along the $z$-axis (i.e., four solutes in each plane). This procedure was done to save computation time and resources and to obtain sufficient statistics. Note that the distances between the four solutes, inserted in each plane, were greater than the cut-off, so that they did not interact with each other during the US simulation. As mentioned above, 72 umbrella windows were used or 12 US simulations were performed to obtain four FEPs, each corresponding to a single pore. Thus, the final FEP of each solute was obtained by averaging over 24 energy profiles (i.e., 4 FEPs $\times$ 6 structures). Similarly, the statistical uncertainties were obtained by calculating the standard deviation between 24 independently built FEPs. In total, 12 US $\times 6$ structures $\times 4$ solutes $\times 3$ model systems $=864$ US simulations were performed to obtain the FEPs. Overall, more than 1000 US simulations were carried out, if we include the test runs performed to select the most appropriate cut-off radius (see above). The US simulations were also carried out in the NPT ensemble, with the same parameters as used in the equilibration simulations. The total US simulation time lasted for $6 \mathrm{~ns}$, of which the last $1 \mathrm{~ns}$ was used to collect the US histograms. All FEPs were constructed using a periodic version of the weighted histogram analysis method (WHAM) [63], by utilizing the gmx wham tool of GROMACS.

\section{Results and Discussion}

As mentioned above, we investigate the permeation of both hydrophilic $\left(\mathrm{H}_{2} \mathrm{O}_{2}\right.$ and $\left.\mathrm{OH}\right)$ and hydrophobic $\left(\mathrm{NO}_{2}\right.$ and $\mathrm{NO})$ RONS through the AQP1 pores of the native (NAT) and oxidized (OXL and OXP) systems. The results of the root mean square displacement (RMSD) of the alpha carbons of AQP1 (see Supplementary Fig. S1a) show that the RMSD in all systems converges after $\sim 60 \mathrm{~ns}$. The equilibrium value fluctuates around $0.32 \mathrm{~nm}$ in the case of NAT and $0.33 \mathrm{~nm}$ in the cases of OXL and OXP, respectively. This slight increase in RMSD indicates that the oxidized systems become slightly more flexible. This can also be deduced from the average pore profile across the AQP1 channels (see Supplementary Fig. S1b), as the fluctuations of the average pore radii in the oxidized systems (OXL and OXP) are slightly higher than in the native (NAT) system. The flexibility in $\mathrm{AQP} 1$, and thereby the higher fluctuations in its pore radii along the $z$-direction, may affect the permeation properties of RONS across AQP1, which is indeed observed in our simulations (see Figure 2). We observe that the minimum pore radius is invariably located at the ar/ $\mathrm{R}$ constriction region. The obtained pore radii are $0.74 \pm 0.06,0.89 \pm 0.17$, and $0.85 \pm 0.10 \AA$ for the NAT, OXL, and OXP systems, respectively. This indicates again that the amino acid residues located in the pores have slightly higher fluctuations after oxidation and can possibly affect the selectivity of the constriction region.

The FEPs of the hydrophilic $\left(\mathrm{H}_{2} \mathrm{O}_{2}\right.$ and $\left.\mathrm{OH}\right)$ and hydrophobic $\left(\mathrm{NO}_{2}\right.$ and NO) RONS across AQP1 of the native (NAT) and oxidized (OXL and OXP) systems are shown in Figure 2. We applied a trapezoidal correction to the FEPs, which enables to compare them with the profiles obtained for the PLB. Information about the trapezoidal correction is given in [31].

As a general trend, all permeants experience barriers when they move from the extracellular aqueous phase to the pore interior. The extent by which oxidation of the lipids (OXL) and the protein (OXP) affects the shape of the FEPs and the barrier heights depends on the permeant. In the case of $\mathrm{H}_{2} \mathrm{O}_{2}$, free energy barriers of $10.6 \pm 2.2 \mathrm{~kJ} / \mathrm{mol}$, $13.9 \pm 1.9 \mathrm{~kJ} / \mathrm{mol}$, and $14.0 \pm 2.7 \mathrm{~kJ} / \mathrm{mol}$ are recorded for NAT, OXL, and OXP, respectively. Oxidation appears to induce a slight increase in the free energy barrier, but the differences are still comparable to the uncertainty limits. In the case of $\mathrm{NO}_{2}$, we also observe a slight increase in the barrier for OXP, but not for OXL. The calculated free energy barriers are $8.9 \pm 1.7 \mathrm{~kJ} / \mathrm{mol}, 8.0 \pm 1.9 \mathrm{~kJ} / \mathrm{mol}$, and $12.7 \pm 1.7 \mathrm{~kJ} / \mathrm{mol}$ for NAT, OXL, and OXP, respectively. Moreover, the local minimum around $-0.6 \mathrm{~nm}$ disappears when oxidation takes place. For $\mathrm{OH}$ and $\mathrm{NO}$, we find either a negligible $(\mathrm{OH})$ or no $(\mathrm{NO})$ effect of oxidation on the energy barrier, but the shape of the FEPs (i.e., local maxima and minima) is affected, as clearly seen for OXL. For OH, the free energy barriers are $7.7 \pm 2.0 \mathrm{~kJ} / \mathrm{mol}, 6.0 \pm 1.9 \mathrm{~kJ} / \mathrm{mol}$, and $8.6 \pm 1.8 \mathrm{~kJ} / \mathrm{mol}$ for NAT, OXL, and OXP, respectively, while for NO, these values are $7.4 \pm 1.4 \mathrm{~kJ} / \mathrm{mol}, 5.5 \pm 1.2 \mathrm{~kJ} / \mathrm{mol}$, and $7.2 \pm 1.6 \mathrm{~kJ} /$ mol for NAT, OXL, and OXP, respectively. Hence, the free energy barriers are somewhat higher for $\mathrm{H}_{2} \mathrm{O}_{2}$ and $\mathrm{NO}_{2}$ than for $\mathrm{OH}$ and $\mathrm{NO}$, which we attribute to the sizes of the species. Important to note is that the FEPs of the hydrophilic RONS $\left(\mathrm{H}_{2} \mathrm{O}_{2}\right.$ and $\left.\mathrm{OH}\right)$ show similarity, i.e., their local minima and maxima are located at around the same $z$ positions (most obviously in the NAT case), and the same applies to the FEPs of the hydrophobic RONS $\left(\mathrm{NO}_{2}\right.$ and $\mathrm{NO}$ ). We should mention here that due to the high reactivity of the $\mathrm{OH}$ radicals, the FEPs obtained from classical nonreactive MD simulations can only provide a partial picture of their behavior in AQP, as these simulations do not account 

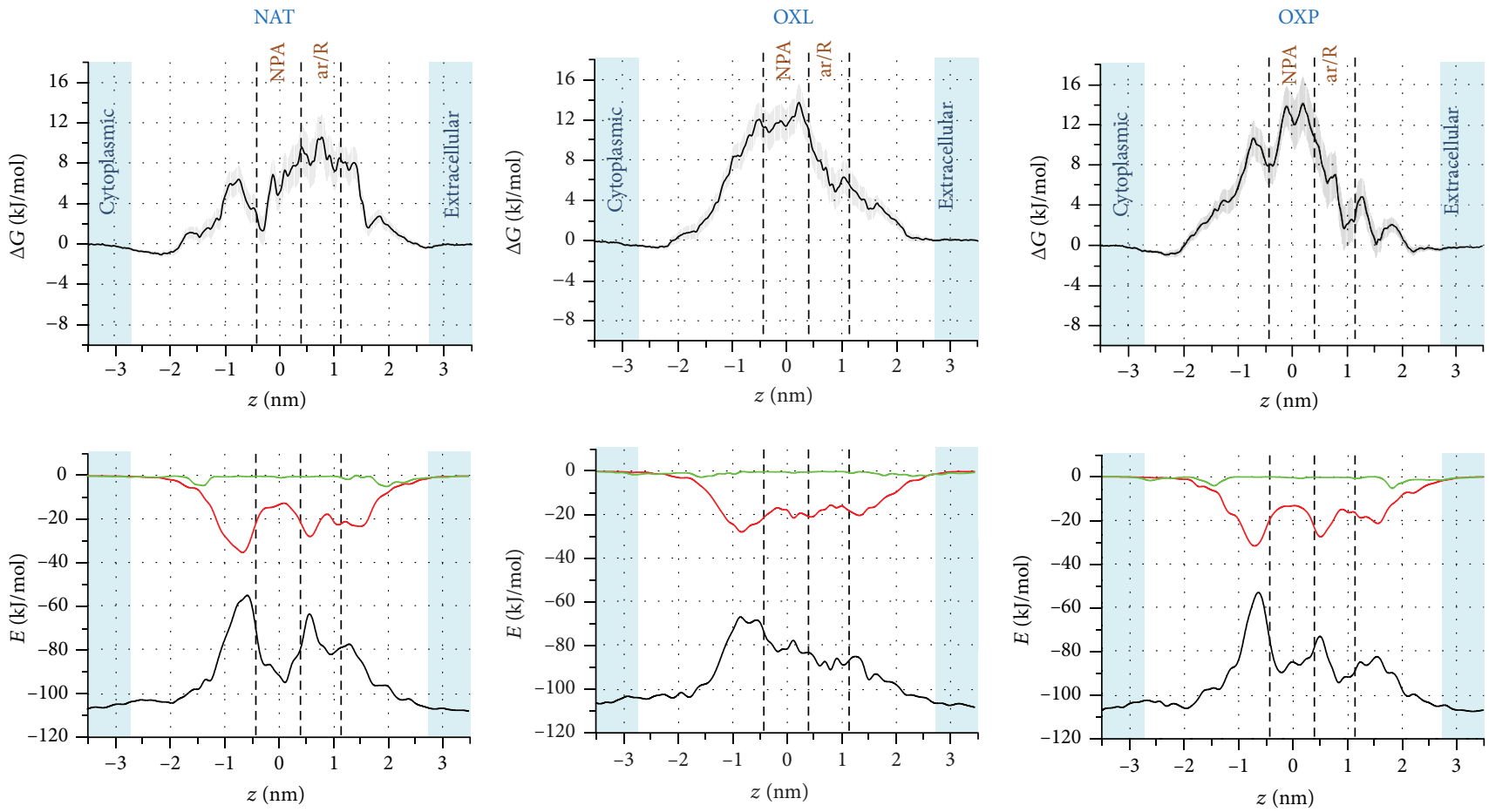

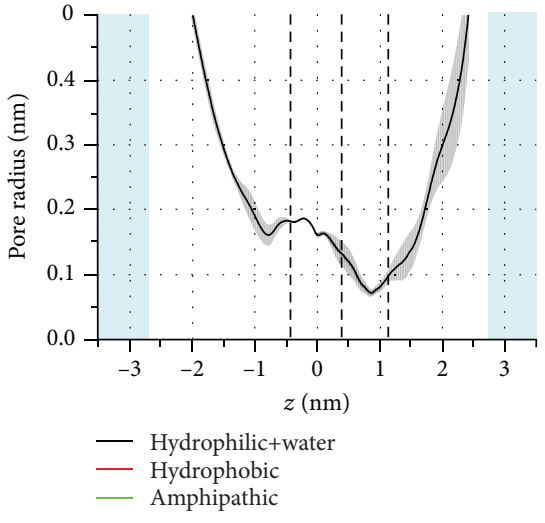

(a)

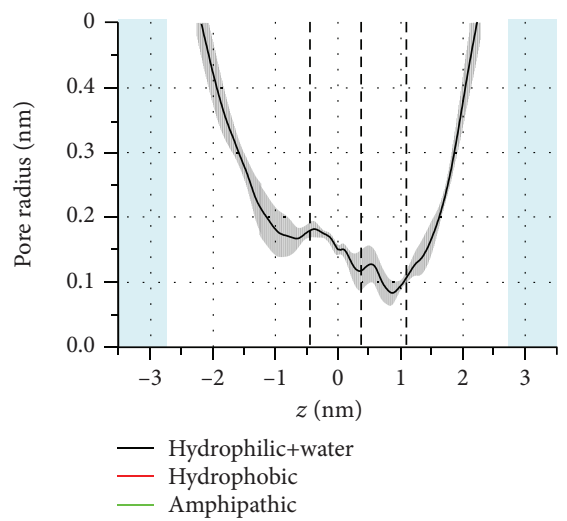

(b)

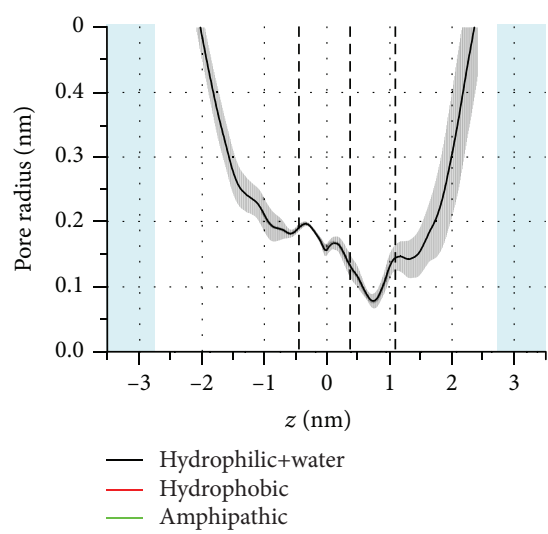

(c)

Figure 3: FEPs (first row) of $\mathrm{H}_{2} \mathrm{O}_{2}$ across the native (NAT (a)) and oxidized model systems (OXL (b) and OXP (c)), together with the nonbonded (i.e., Coulomb+van der Waals) interaction energy (second row) and pore radius (third row) profiles. The nonbonded interaction energies between $\mathrm{H}_{2} \mathrm{O}_{2}$ and the hydrophilic residues+water, the hydrophobic, and the amphipathic residues are shown in black, red, and green, respectively.

for chemical reactions. Nevertheless, it may still be helpful to know the classical free energy barriers that $\mathrm{OH}$ radicals need to overcome in order to have access to the pore interior. A more detailed discussion about the importance of the FEPs of $\mathrm{OH}$ is given in the Supplementary Information.

We propose a qualitative explanation for the FEPs by focusing on the nonbonded (i.e., Coulomb+van der Waals) interaction energies and pore radius profiles, as illustrated in Figures 3 and 4 for $\mathrm{H}_{2} \mathrm{O}_{2}$ (hydrophilic) and $\mathrm{NO}_{2}$ (hydrophobic), respectively. The same analysis is done for the $\mathrm{OH}$ and NO radicals and presented in Supplementary Information (Figs. S2 and S3).

The nonbonded interaction energies (NBEs) along the $z$-axis are calculated between $\mathrm{H}_{2} \mathrm{O}_{2}$ and the hydrophilic, hydrophobic, and amphipathic amino acid residues of
AQP1, as well as the water molecules located inside and outside of the AQP1 pores. To facilitate the explanation, we combine the NBE profiles for the hydrophilic residues with water (i.e., hydrophilic+water).

In all cases (i.e., NAT, OXL, and OXP), the NBE of $\mathrm{H}_{2} \mathrm{O}_{2}$ with the hydrophilic residues+water is higher (i.e., more negative) than with the hydrophobic and amphipathic residues. This indicates that the hydrophilic residues+water have a larger contribution for the interactions. Indeed, $\mathrm{H}_{2} \mathrm{O}_{2}$, being itself a hydrophilic species, strongly interacts with the hydrophilic residues and especially with water, causing the most negative energy values to be found in the extracellular and cytoplasmic aqueous regions. Furthermore, in all cases, the NBEs of $\mathrm{H}_{2} \mathrm{O}_{2}$ with the amphipathic residues are close to zero. 

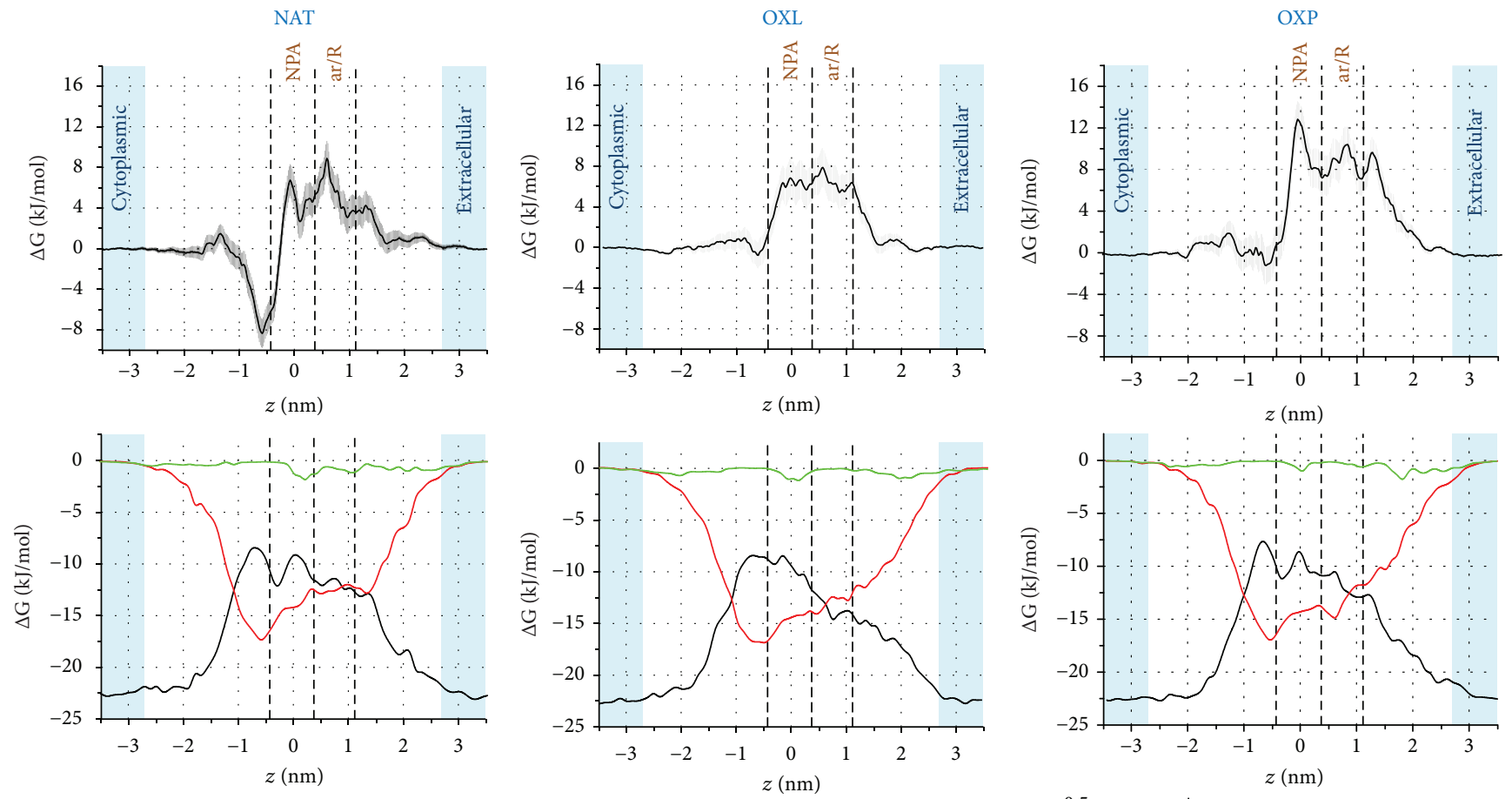

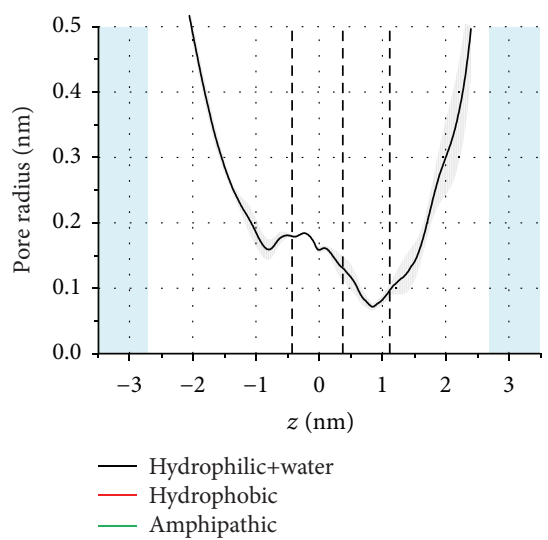

(a)

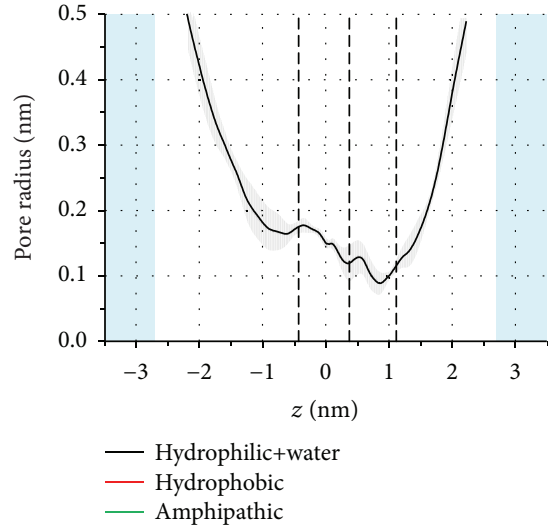

(b)

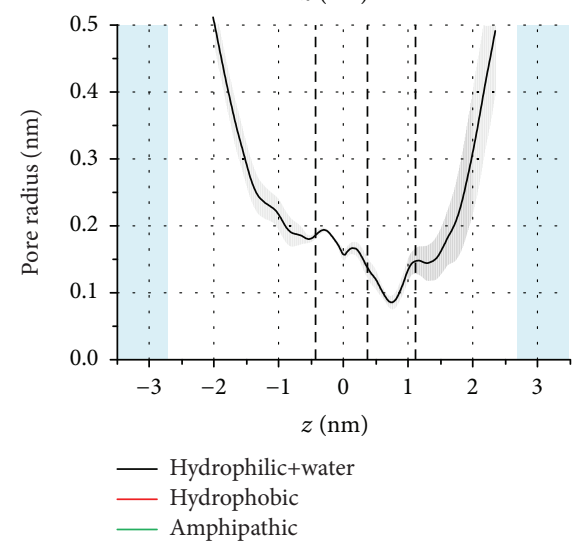

(c)

FIGURE 4: FEPs (first row) of $\mathrm{NO}_{2}$ across the native (NAT (a)) and oxidized model systems (OXL (b) and OXP (c)), together with the nonbonded (i.e., Coulomb+van der Waals) interaction energy (second row) and pore radius (third row) profiles. The nonbonded interaction energies between $\mathrm{NO}_{2}$ and the hydrophilic residues+water, the hydrophobic, and the amphipathic residues are shown in black, red, and green, respectively.

In the NAT system (Figure 3(a)), the NBE of $\mathrm{H}_{2} \mathrm{O}_{2}$ with the hydrophilic residues+water decreases (i.e., becomes less negative) when $\mathrm{H}_{2} \mathrm{O}_{2}$ penetrates from the extracellular region to the pore interior, leading to an increase in the free energy barrier (first row of Figure 3). This is only partially compensated by the increase of the NBE with the hydrophobic residues, resulting in an overall weakening of the interaction energy, which might in turn explain the increase of the free energy. The confinement experienced by $\mathrm{H}_{2} \mathrm{O}_{2}$ inside the channel, especially in the ar/ $\mathrm{R}$ constriction region, creates an entropic penalty that reflects on the free energy value. The maximum free energy barrier is observed in the ar/R region, which corresponds to the minimum pore radius (third row in Figure 3). Close to the end of the ar/ $\mathrm{R}$ region $(z \approx 0-0.5 \mathrm{~nm})$, the NBE of $\mathrm{H}_{2} \mathrm{O}_{2}$ with the hydrophilic residues+water clearly increases (i.e., becomes more negative), whereas it decreases for the hydrophobic residues, resulting in an overall drop of the free energy in the NPA region. This is also attributed to a somewhat larger pore radius in the NPA region, so the interaction of $\mathrm{H}_{2} \mathrm{O}_{2}$ with the water molecules in the pore becomes stronger and the entropic penalty lower. Beyond the NPA region $(z \approx-0.9 \mathrm{~nm})$, the pore radius slightly drops and the NBE of $\mathrm{H}_{2} \mathrm{O}_{2}$ with the hydrophilic residues+water becomes again weaker, while the NBE with the hydrophobic residues becomes stronger, again leading to an increase in the free energy. The changes in the NBEs are more pronounced here than in the $\mathrm{ar} / \mathrm{R}$ region, showing stronger interaction of $\mathrm{H}_{2} \mathrm{O}_{2}$ with the hydrophobic residues and weaker interaction with the hydrophilic residues+water. Therefore, the barrier increase at $z \approx-0.9 \mathrm{~nm}$ is not as high as in the ar/R region.

Similar interpretations of the FEPs can be made for OXL (Figure 3(b)) and OXP (Figure 3(c)). The only difference is 
that the pore radii in the OXL and OXP systems are somewhat larger in the ar/R constriction region compared to that in the NAT system (see above) and have higher fluctuations along the channel. This in turn affects the NBE of $\mathrm{H}_{2} \mathrm{O}_{2}$ (or other permeants in general), thereby influencing the FEP. In the OXL case (Figure 3(b)), the $\mathrm{NBE}$ of $\mathrm{H}_{2} \mathrm{O}_{2}$ with the hydrophilic residues+water decreases more or less linearly (i.e., becomes less negative) from the extracellular aqueous phase till $z \approx-0.9 \mathrm{~nm}$, while the NBE with the hydrophobic residues increases (i.e., becomes more negative) up to this position, being more or less constant in the ar/R and NPA regions. The drop in the NBE with the hydrophilic residues+water and the confinement lead to the free energy barrier, which reaches its maximum in the NPA region at around $z=0.0 \mathrm{~nm}$. Beyond this position, the NBE with the hydrophobic residues rises (becomes more negative), and therefore, the free energy starts to decrease. From around $z=-1.0 \mathrm{~nm}$ till the cytoplasmic region, the NBE with the hydrophilic residues+water becomes very strong, resulting in a drop in the free energy. Likewise, the FEP of the OXP case (Figure 3(c)) can also be explained from the NBEs of $\mathrm{H}_{2} \mathrm{O}_{2}$ with the hydrophilic residues+water and with the hydrophobic residues, just like in the NAT case.

It is interesting to note that the oxidation of $\mathrm{Cys}_{191}$ to sulfenic acid near the ar/R constriction turns this region more hydrophilic. As expected, the hydrophilic interactions of $\mathrm{H}_{2} \mathrm{O}_{2}$ in this region become stronger and the free energy value decreases. The free energy barrier, which was originally located at the ar/R constriction in the NAT system, is shifted to the other side of the channel, beyond the NPA region, in OXP. We speculate that the sulfenic acid group may affect the delicate interaction balance that maintains the bipolar orientation of the water molecules inside the channel (cf. [31]). A less organized water file inside the channel can possibly explain why the oxidation of $\mathrm{Cys}_{191}$ is able to affect the free energy in distal regions inside the channel. Although a similar increase of the free energy barrier was observed in OXP and OXL, the mechanisms are probably different. Both the free energy and NBE profiles are much less structured in OXL as compared to OXP. It is well known that lipid oxidation decreases the membrane thickness [64-66], which in turn has an impact on the AQP properties [67]. We hypothesize that the change in membrane thickness may disturb the AQP structure and impact the water file organization in a similar fashion as in the case of $\mathrm{Cys}_{191}$ oxidation. It is tempting to draw a parallel between the increase in the free energy barrier witnessed upon oxidation and the alleged oxidative gating of AQPs [33-35].

We do not provide a detailed explanation for the FEP of the hydrophobic $\mathrm{NO}_{2}$ (see Figure 4), as the analysis is very similar as for $\mathrm{H}_{2} \mathrm{O}_{2}$. We concentrate mainly on the difference in the NBE profiles of $\mathrm{NO}_{2}$ and $\mathrm{H}_{2} \mathrm{O}_{2}$, because they help to explain the observed FEPs. As is clear from Figure 4, the strongest NBE is observed again in the extracellular and cytoplasmic aqueous regions (i.e., around $-23 \mathrm{~kJ} / \mathrm{mol}$, see black curve in the second row), which corresponds to the interaction between $\mathrm{NO}_{2}$ and water. Note that this energy is $\sim 5$ times lower than the energy obtained for $\mathrm{H}_{2} \mathrm{O}_{2}$ in these regions, due to the hydrophobicity of $\mathrm{NO}_{2}$.
On the other hand, the maximum NBE with the hydrophobic residues is around $-17.5 \mathrm{~kJ} / \mathrm{mol}$ (see red curve in second row of Figure 4), which is only $\sim 2$ times lower than the $\mathrm{NBE}$ of $\mathrm{H}_{2} \mathrm{O}_{2}$ with the hydrophobic residues (cf. Figure 3). This indicates that although the $\mathrm{NBE}$ of $\mathrm{H}_{2} \mathrm{O}_{2}$ is stronger with both the hydrophilic residues+water and the hydrophobic residues, compared to the $\mathrm{NBE}$ of $\mathrm{NO}_{2}$, the interaction of $\mathrm{NO}_{2}$ with the hydrophobic residues is relatively stronger, as expected based on its hydrophobicity. Indeed, the deep minimum of the FEP observed in the NAT case (i.e., at around $z=-0.6 \mathrm{~nm}$, see Figure $4(\mathrm{a}))$ is due to the stronger interaction of $\mathrm{NO}_{2}$ with the hydrophobic residues compared to the interaction with the hydrophilic residues+water. This minimum in the FEP disappears in the case of OXL and OXP (Figures 4(b) and 4(c)), most likely due to the higher fluctuations in the pore radius profiles. The other characteristics of the FEPs can be explained as was done above for $\mathrm{H}_{2} \mathrm{O}_{2}$. A similar analysis can also be made for the FEPs of the $\mathrm{OH}$ and NO radicals (see Figs. S2 and S3).

Thus, in general, the calculated NBE and pore radius profiles help to explain the influence of the different types of amino acid residues and of water molecules on the FEPs of RONS. The NBEs of the hydrophilic RONS are much stronger than those of the hydrophobic species (cf. Figures 3 and 4 , as well as Figs. S2 and S3). For the hydrophilic species $\left(\mathrm{H}_{2} \mathrm{O}_{2}\right.$ and $\left.\mathrm{OH}\right)$, the NBE contributions of the hydrophilic residues+water to the overall interactions are higher than the contributions of the hydrophobic residues. Analogously, for the hydrophobic species $\left(\mathrm{NO}_{2}\right.$ and $\left.\mathrm{NO}\right)$, the $\mathrm{NBE}$ contributions of the hydrophobic residues to the overall energy are higher than the contributions of the hydrophilic residues+water.

It should be mentioned that the central cavity of the AQP tetramer (see Figure 1(a)) may also play a role in the transport of RONS into the cell interior. However, we do not consider the contribution of the central cavity because our previous studies showed that the obtained free energy barriers of the hydrophilic species $\left(\mathrm{OH}\right.$ and $\left.\mathrm{H}_{2} \mathrm{O}_{2}\right)$ through the central cavity are much higher than through individual pores $[31,32]$. These barriers are either similar or higher than those calculated at the native PLB. Thus, it is highly conceivable that the hydrophilic species most probably permeate across the pores, even when lipid or protein oxidation takes place, as they experience lower free energy barriers. The hydrophobic species ( $\mathrm{NO}$ and $\mathrm{NO}_{2}$ ), on the other hand, most likely prefer the transport across the PLB, as they experience significantly low permeation barriers (i.e., $\sim 1 \mathrm{~kJ} / \mathrm{mol}$ ) [55]. These barriers are negligible compared to those through AQP pores obtained in this study using both lipid and protein oxidation.

Finally, it should also be mentioned that real cell membranes are vastly more complex than the simple membrane models considered here. Cell membranes are made of a complex mixture of lipids and embedded membrane proteins, which are able to segregate and form heterogeneous domains at the nanometer length scale. The expression of membrane proteins such as AQPs is subject to intricate regulation mechanisms which are also expected to play a role in the cellular response to oxidative stress. With that in mind, the effects 
of oxidative stress on AQP permeability, as unveiled by our simulations, should be viewed as part of a larger and more complex biochemical machinery.

\section{Conclusions}

The aim of our study was to better understand the permeation process of both hydrophilic $\left(\mathrm{H}_{2} \mathrm{O}_{2}\right.$ and $\left.\mathrm{OH}\right)$ and hydrophobic $\left(\mathrm{NO}_{2}\right.$ and $\left.\mathrm{NO}\right)$ RONS across the pores of AQP1, which is a transmembrane protein, as well as the effect of both lipid and protein oxidation, which can be induced by these RONS.

Our results showed that oxidation of a single Cys ${ }_{191}$ residue in each pore of AQP1 (i.e., protein oxidation) has almost the same effect on the free energy barrier of $\mathrm{H}_{2} \mathrm{O}_{2}$ as $50 \%$ lipid oxidation in the PLB. For $\mathrm{OH}$ and NO, the barrier in case of protein oxidation is even slightly higher, while it is clearly higher in the case of $\mathrm{NO}_{2}$ compared to $50 \%$ lipid oxidation. Overall, both lipid and protein oxidation influences the shape of the FEPs of all RONS, as well as the barrier heights for $\mathrm{H}_{2} \mathrm{O}_{2}$ and $\mathrm{NO}_{2}$. In general, the free energy barriers are somewhat higher for $\mathrm{H}_{2} \mathrm{O}_{2}$ and $\mathrm{NO}_{2}$ than for $\mathrm{OH}$ and $\mathrm{NO}$, which we attributed to the sizes of these species.

To explain the FEPs, we studied the NBEs of the RONS with the hydrophilic and hydrophobic amino acid residues and water, found both inside and outside of the pores, as well as the pore radius profiles. They help to explain the shape of the FEPs, by showing the amino acid residues and water molecules involved in the permeation process.

Our simulation results indicate that, overall, oxidation does not strongly affect the transport of RONS through AQP. However, we note that regular MD simulations are unable to capture large-scale protein structural changes that happen beyond the multimicrosecond time domain. As the barrier for transport of hydrophilic RONS through AQP (both native and oxidized) is quite low $(\sim 6-12 \mathrm{~kJ} / \mathrm{mol})$, we suggest that these species (i.e., $\mathrm{OH}$ and especially $\mathrm{H}_{2} \mathrm{O}_{2}$ ) will enter the cell through AQPs, as they experience a clearly higher barrier through the PLB $(\sim 15-30 \mathrm{~kJ} / \mathrm{mol})[32,53$, $65]$, especially in the native (i.e., unstirred) lipid bilayer. On the other hand, the hydrophobic RONS (i.e., $\mathrm{NO}, \mathrm{NO}_{2}$, but also others, like $\mathrm{O}_{2}$ ) most likely permeate through the PLB, where lipid (per)oxidation can take place, as their barrier through AQP is higher (i.e., $\sim 5-12 \mathrm{~kJ} / \mathrm{mol}$ vs. $\sim 1 \mathrm{~kJ} / \mathrm{mol}$ for the PLB [55]).

Our results provide molecular level insight into the processes of RONS permeation through the membrane and specifically into the role of AQP, which is often more expressed in cancer cells. This may help to improve our understanding on the selective rise of RONS observed in cancer cells, shedding light on the selective anticancer mechanism of CAP.

\section{Data Availability}

The data used to support the findings of this study are available from the corresponding author upon request.

\section{Conflicts of Interest}

The authors declare that there is no conflict of interest regarding the publication of this article.

\section{Acknowledgments}

The authors acknowledge the Turing HPC infrastructure at the CalcUA core facility of the University of Antwerp (UA), a division of the Flemish Supercomputer Center VSC, funded by the Hercules Foundation, the Flemish Government (department EWI), and the UA, where all computational work was performed. M.Y. gratefully acknowledges Dr. U. Khalilov for the fruitful discussions. This work was financially supported by the Research Foundation Flanders (FWO) (grant number 1200219N).

\section{Supplementary Materials}

Supplementary Information: further details about the chosen oxidized model systems and the three supplementary figures. (Supplementary Materials)

\section{References}

[1] M. Keidar, R. Walk, A. Shashurin et al., "Cold plasma selectivity and the possibility of a paradigm shift in cancer therapy," British Journal of Cancer, vol. 105, no. 9, pp. 1295-1301, 2011.

[2] M. Wang, B. Holmes, X. Cheng, W. Zhu, M. Keidar, and L. G. Zhang, "Cold atmospheric plasma for selectively ablating metastatic breast cancer cells," PloS One, vol. 8, no. 9, article e73741, 2013

[3] F. Utsumi, H. Kajiyama, K. Nakamura, H. Tanaka, M. Hori, and F. Kikkawa, "Selective cytotoxicity of indirect nonequilibrium atmospheric pressure plasma against ovarian clear-cell carcinoma," SpringerPlus, vol. 3, no. 1, p. 398, 2014.

[4] S. J. Kim and T. H. Chung, "Cold atmospheric plasma jetgenerated RONS and their selective effects on normal and carcinoma cells," Scientific Reports, vol. 6, no. 1, 2016.

[5] H.-R. Metelmann, C. Seebauer, V. Miller et al., "Clinical experience with cold plasma in the treatment of locally advanced head and neck cancer," Clinical Plasma Medicine, vol. 9, pp. 6-13, 2018.

[6] E. A. Ratovitski, X. Cheng, D. Yan et al., "Anti-cancer therapies of 21st century: novel approach to treat human cancers using cold atmospheric plasma," Plasma Processes and Polymers, vol. 11, no. 12, pp. 1128-1137, 2014.

[7] J. Y. Kim, J. Ballato, P. Foy et al., "Apoptosis of lung carcinoma cells induced by a flexible optical fiber-based cold microplasma," Biosensors and Bioelectronics, vol. 28, no. 1, pp. 333-338, 2011.

[8] M. Ishaq, M. D. M. Evans, and K. (. K.). Ostrikov, "Atmospheric pressure gas plasma-induced colorectal cancer cell death is mediated by Nox2-ASK1 apoptosis pathways and oxidative stress is mitigated by Srx-Nrf2 anti-oxidant system," Biochimica et Biophysica Acta (BBA)-Molecular Cell Research, vol. 1843, no. 12, pp. 2827-2837, 2014.

[9] M. Ishaq, S. Kumar, H. Varinli et al., "Atmospheric gas plasma-induced ROS production activates TNF-ASK1 pathway for the induction of melanoma cancer cell apoptosis," 
Molecular Biology of the Cell, vol. 25, no. 9, pp. 1523-1531, 2014.

[10] S. Ja Kim, H. Min Joh, and T. H. Chung, "Production of intracellular reactive oxygen species and change of cell viability induced by atmospheric pressure plasma in normal and cancer cells," Applied Physics Letters, vol. 103, no. 15, article 153705, 2013.

[11] D. B. Graves, "Reactive species from cold atmospheric plasma: implications for cancer therapy," Plasma Processes and Polymers, vol. 11, no. 12, pp. 1120-1127, 2014.

[12] G. Bauer, "Increasing the endogenous NO level causes catalase inactivation and reactivation of intercellular apoptosis signaling specifically in tumor cells," Redox Biology, vol. 6, pp. 353-371, 2015.

[13] D. Yan, A. Talbot, N. Nourmohammadi, J. H. Sherman, $\mathrm{X}$. Cheng, and M. Keidar, "Toward understanding the selective anticancer capacity of cold atmospheric plasma-a model based on aquaporins Biointerphases," Biointerphases, vol. 10, no. 4, article 040801, 2015.

[14] E. J. Szili, S.-H. Hong, and R. D. Short, “On the effect of serum on the transport of reactive oxygen species across phospholipid membranes," Biointerphases, vol. 10, no. 2, article 029511, 2015.

[15] J. Van der Paal, E. C. Neyts, C. C. Verlackt, and A. Bogaerts, "Effect of lipid peroxidation on membrane permeability of cancer and normal cells subjected to oxidative stress," Chemical Science, vol. 7, no. 1, pp. 489-498, 2016.

[16] E. Kruse, N. Uehlein, and R. Kaldenhoff, "The aquaporins," Genome biology, vol. 7, no. 2, p. 206, 2006.

[17] B. Wu and E. Beitz, "Aquaporins with selectivity for unconventional permeants," Cellular and molecular life sciences, vol. 64, no. 18, pp. 2413-2421, 2007.

[18] A. Verkman, M. Hara-Chikuma, and M. C. Papadopoulos, "Aquaporins-new players in cancer biology," Journal of Molecular Medicine, vol. 86, no. 5, pp. 523-529, 2008.

[19] D. Yan, H. Xiao, W. Zhu et al., "The role of aquaporins in the anti-glioblastoma capacity of the cold plasma-stimulated medium," Journal of Physics D: Applied Physics, vol. 50, no. 5, article 055401, 2017.

[20] E. W. Miller, B. C. Dickinson, and C. J. Chang, "Aquaporin-3 mediates hydrogen peroxide uptake to regulate downstream intracellular signaling," Proceedings of the National Academy of Sciences, vol. 107, no. 36, pp. 15681-15686, 2010.

[21] Y. Wang and E. Tajkhorshid, "Molecular mechanisms of conduction and selectivity in aquaporin water channels," The Journal of nutrition, vol. 137, no. 6, pp. 1509S-1515S, 2007.

[22] F. Zhu, E. Tajkhorshid, and K. Schulten, "Theory and simulation of water permeation in aquaporin-1," Biophysical Journal, vol. 86, no. 1, pp. 50-57, 2004.

[23] Y. Cui and D. A. Bastien, "Water transport in human aquaporin-4: molecular dynamics (MD) simulations," Biochemical and biophysical research communications, vol. 412, no. 4, pp. 654-659, 2011.

[24] B. L. de Groot and H. Grubmüller, "Water permeation across biological membranes: mechanism and dynamics of aquaporin-1 and GlpF," Science, vol. 294, no. 5550, pp. 2353-2357, 2001.

[25] Y. Hirano, N. Okimoto, I. Kadohira, M. Suematsu, K. Yasuoka, and M. Yasui, "Molecular mechanisms of how mercury inhibits water permeation through aquaporin-1: understand- ing by molecular dynamics simulation," Biophysical journal, vol. 98, no. 8, pp. 1512-1519, 2010.

[26] M. Ø. Jensen, E. Tajkhorshid, and K. Schulten, "The mechanism of glycerol conduction in aquaglyceroporins," Structure, vol. 9, no. 11, pp. 1083-1093, 2001.

[27] J. S. Hub and B. L. de Groot, "Does $\mathrm{CO}_{2}$ permeate through aquaporin-1?," Biophysical Journal, vol. 91, no. 3, pp. $842-$ 848, 2006.

[28] J. S. Hub and B. L. de Groot, "Mechanism of selectivity in aquaporins and aquaglyceroporins," Proceedings of the National Academy of Sciences, vol. 105, no. 4, pp. 1198-1203, 2008.

[29] C. Aponte-Santamaría, J. S. Hub, and B. L. de Groot, "Dynamics and energetics of solute permeation through the Plasmodium falciparum aquaglyceroporin," Physical Chemistry Chemical Physics, vol. 12, no. 35, pp. 1024610254, 2010.

[30] Y. Wang and E. Tajkhorshid, "Nitric oxide conduction by the brain aquaporin AQP4," Proteins: Structure, Function, and Bioinformatics, vol. 78, pp. 661-670, 2010.

[31] R. M. Cordeiro, "Molecular dynamics simulations of the transport of reactive oxygen species by mammalian and plant aquaporins," Biochimica et Biophysica Acta (BBA)-General Subjects, vol. 1850, no. 9, pp. 1786-1794, 2015.

[32] M. Yusupov, D. Yan, R. M. Cordeiro, and A. Bogaerts, "Atomic scale simulation of $\mathrm{H}_{2} \mathrm{O}_{2}$ permeation through aquaporin: toward the understanding of plasma cancer treatment," Journal of Physics D: Applied Physics, vol. 51, no. 12, article 125401, 2018.

[33] Q. Ye and E. Steudle, "Oxidative gating of water channels (aquaporins) in corn roots," Plant, Cell \& Environment, vol. 29, no. 4, pp. 459-470, 2006.

[34] Y. X. Kim and E. Steudle, "Gating of aquaporins by light and reactive oxygen species in leaf parenchyma cells of the midrib of Zea mays," Journal of Experimental Botany, vol. 60, no. 2, pp. 547-556, 2008.

[35] T. Henzler, Q. Ye, and E. Steudle, "Oxidative gating of water channels (aquaporins) in Chara by hydroxyl radicals," Plant, Cell \& Environment, vol. 27, no. 9, pp. 1184-1195, 2004.

[36] D. B. Graves, "The emerging role of reactive oxygen and nitrogen species in redox biology and some implications for plasma applications to medicine and biology," Journal of Physics D: Applied Physics, vol. 45, no. 26, article 263001, 2012.

[37] H. Sui, B.-G. Han, J. K. Lee, P. Walian, and B. K. Jap, "Structural basis of water-specific transport through the AQP1 water channel," Nature, vol. 414, no. 6866, pp. 872-878, 2001.

[38] G. Van Meer, D. R. Voelker, and G. W. Feigenson, "Membrane lipids: where they are and how they behave," Nature reviews Molecular cell biology, vol. 9, no. 2, pp. 112-124, 2008.

[39] A. Reis, M. Domingues, F. M. Amado, A. Ferrer-Correia, and P. Domingues, "Separation of peroxidation products of diacyl-phosphatidylcholines by reversed-phase liquid chromatography-mass spectrometry," Biomedical Chromatography, vol. 19, no. 2, pp. 129-137, 2005.

[40] G. Xu and M. R. Chance, "Hydroxyl radical-mediated modification of proteins as probes for structural proteomics," Chemical Reviews, vol. 107, no. 8, pp. 3514-3543, 2007.

[41] M. Ø. Jensen, U. Röthlisberger, and C. Rovira, "Hydroxide and Proton Migration in Aquaporins," Biophysical Journal, vol. 89, no. 3, pp. 1744-1759, 2005. 
[42] P. Agre, "Aquaporin water channels (Nobel lecture)," Angewandte Chemie International Edition, vol. 43, no. 33, pp. 4278-4290, 2004.

[43] K. Murata, K. Mitsuoka, T. Hirai et al., "Structural determinants of water permeation through aquaporin-1," Nature, vol. 407, no. 6804, pp. 599-605, 2000.

[44] S. Jo, T. Kim, V. G. Iyer, and W. Im, "CHARMM-GUI: a webbased graphical user interface for CHARMM," Journal of computational chemistry, vol. 29, no. 11, pp. 1859-1865, 2008.

[45] E. L. Wu, X. Cheng, S. Jo et al., "CHARMM-GUI membrane builder toward realistic biological membrane simulations," Journal of computational chemistry, vol. 35, no. 27, pp. 19972004, 2014.

[46] M. A. Lomize, I. D. Pogozheva, H. Joo, H. I. Mosberg, and A. L. Lomize, "OPM database and PPM web server: resources for positioning of proteins in membranes," Nucleic acids research, vol. 40, no. D1, pp. D370-D376, 2011.

[47] L. Martínez, R. Andrade, E. G. Birgin, and J. M. Martínez, "PACKMOL: a package for building initial configurations for molecular dynamics simulations," Journal of computational chemistry, vol. 30, no. 13, pp. 2157-2164, 2009.

[48] C. Margreitter, D. Petrov, and B. Zagrovic, "Vienna-PTM web server: a toolkit for MD simulations of protein posttranslational modifications," Nucleic acids research, vol. 41, no. W1, pp. W422-W426, 2013.

[49] W. G. Hoover, "Canonical dynamics: equilibrium phase-space distributions," Physical review A, vol. 31, no. 3, pp. 1695-1697, 1985.

[50] M. Parrinello and A. Rahman, "Polymorphic transitions in single crystals: a new molecular dynamics method," Journal of Applied physics, vol. 52, no. 12, pp. 7182-7190, 1981.

[51] N. Schmid, A. P. Eichenberger, A. Choutko et al., "Definition and testing of the GROMOS force-field versions 54A7 and 54B7," European biophysics journal, vol. 40, no. 7, pp. 843856, 2011.

[52] H. J. Berendsen, J. P. Postma, W. F. van Gunsteren, and J. Hermans, "Interaction Models for Water in Relation to Protein Hydration," in Intermolecular Forces, B. Pullman, Ed., vol. 14 of The Jerusalem Symposia on Quantum Chemistry and Biochemistry, pp. 331-342, Springer, Dordrecht, 1981.

[53] R. M. Cordeiro, "Reactive oxygen species at phospholipid bilayers: distribution, mobility and permeation," Biochimica et Biophysica Acta (BBA)-Biomembranes, vol. 1838, no. 1, Part B, pp. 438-444, 2014.

[54] R. M. Cordeiro, "Reactive oxygen and nitrogen species at phospholipid bilayers: peroxynitrous acid and its homolysis products," The Journal of Physical Chemistry B, vol. 122, no. 34, pp. 8211-8219, 2018.

[55] J. Razzokov, M. Yusupov, R. M. Cordeiro, and A. Bogaerts, "Atomic scale understanding of the permeation of plasma species across native and oxidized membranes," Journal of Physics D: Applied Physics, vol. 51, no. 36, article 365203, 2018.

[56] J. Wong-Ekkabut, Z. Xu, W. Triampo, I.-M. Tang, D. P. Tieleman, and L. Monticelli, "Effect of lipid peroxidation on the properties of lipid bilayers: a molecular dynamics study," Biophysical journal, vol. 93, no. 12, pp. 4225-4236, 2007.

[57] D. Petrov, C. Margreitter, M. Grandits, C. Oostenbrink, and B. Zagrovic, "A systematic framework for molecular dynamics simulations of protein post-translational modifications," PLoS computational biology, vol. 9, no. 7, article e1003154, 2013.
[58] N. Kučerka, M.-P. Nieh, and J. Katsaras, "Fluid phase lipid areas and bilayer thicknesses of commonly used phosphatidylcholines as a function of temperature," Biochimica et Biophysica Acta (BBA)-Biomembranes, vol. 1808, no. 11, pp. 2761-2771, 2011.

[59] M. J. Abraham, T. Murtola, R. Schulz et al., "GROMACS: high performance molecular simulations through multi-level parallelism from laptops to supercomputers," SoftwareX, vol. 1-2, pp. 19-25, 2015.

[60] W. Humphrey, A. Dalke, and K. Schulten, "VMD: visual molecular dynamics," Journal of Molecular Graphics, vol. 14, no. 1, pp. 33-38, 1996.

[61] O. S. Smart, J. G. Neduvelil, X. Wang, B. A. Wallace, and M. S. P. Sansom, "HOLE: a program for the analysis of the pore dimensions of ion channel structural models," Journal of Molecular Graphics, vol. 14, no. 6, pp. 354-360, 1996.

[62] J. Kästner, "Umbrella sampling," Wiley Interdisciplinary Reviews: Computational Molecular Science, vol. 1, pp. 932942, 2011.

[63] J. S. Hub, B. L. de Groot, and D. van der Spoel, "g_wham-a free weighted histogram analysis implementation including robust error and autocorrelation estimates," Journal of Chemical Theory and Computation, vol. 6, no. 12, pp. 3713-3720, 2010.

[64] A. J. Neto and R. M. Cordeiro, "Molecular simulations of the effects of phospholipid and cholesterol peroxidation on lipid membrane properties," Biochimica et Biophysica Acta (BBA) - Biomembranes, vol. 1858, no. 9, pp. 2191-2198, 2016.

[65] M. Yusupov, J. Van der Paal, E. Neyts, and A. Bogaerts, "Synergistic effect of electric field and lipid oxidation on the permeability of cell membranes," Biochimica et Biophysica Acta (BBA) - General Subjects, vol. 1861, no. 4, pp. 839-847, 2017.

[66] M. Yusupov, K. Wende, S. Kupsch, E. Neyts, S. Reuter, and A. Bogaerts, "Effect of head group and lipid tail oxidation in the cell membrane revealed through integrated simulations and experiments," Scientific Reports, vol. 7, no. 1, p. 5761, 2017.

[67] M. Ø. Jensen and O. G. Mouritsen, "Lipids do influence protein function-the hydrophobic matching hypothesis revisited," Biochimica et Biophysica Acta (BBA)-Biomembranes, vol. 1666, no. 1-2, pp. 205-226, 2004. 


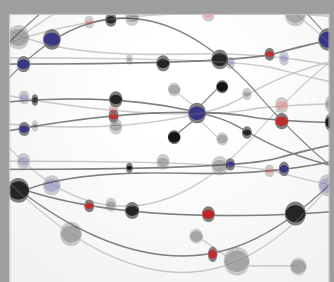

The Scientific World Journal
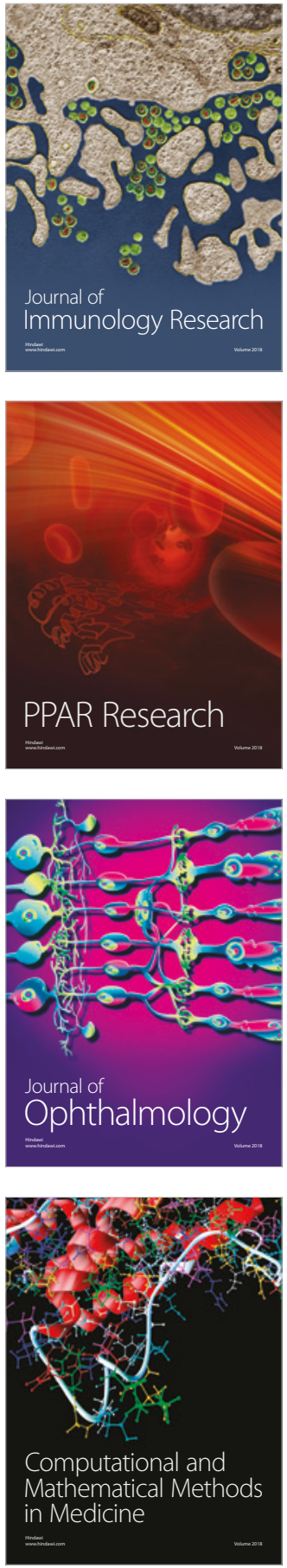

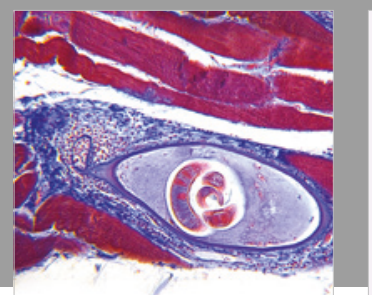

Gastroenterology Research and Practice

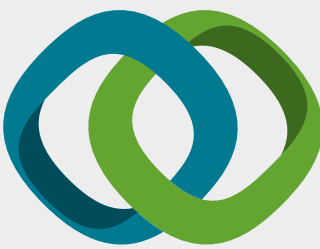

\section{Hindawi}

Submit your manuscripts at

www.hindawi.com
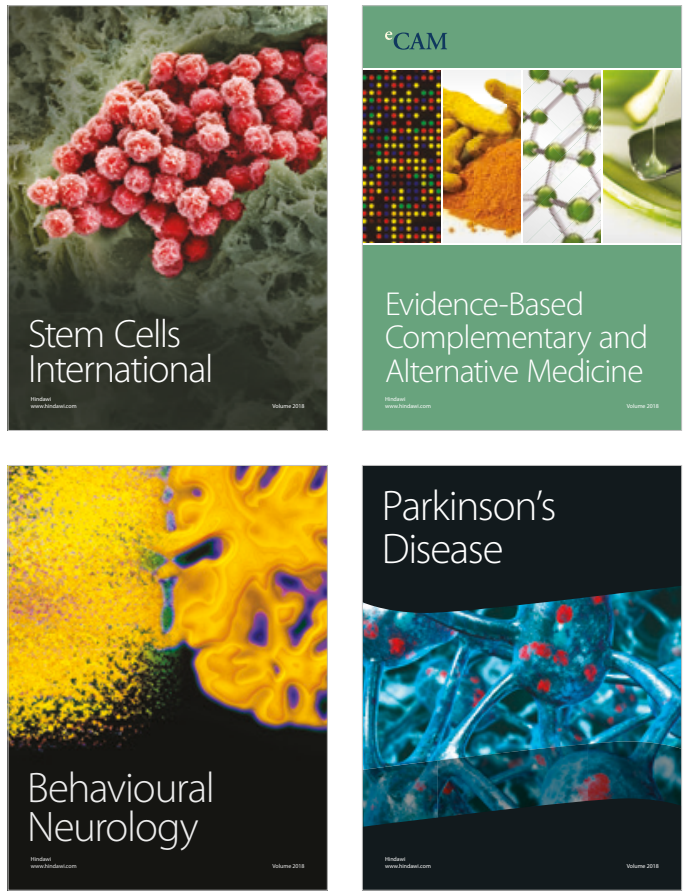

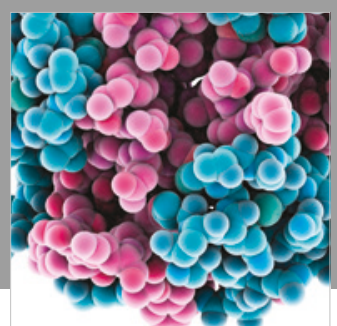

ournal of

Diabetes Research

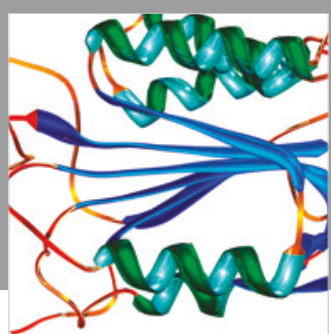

Disease Markers
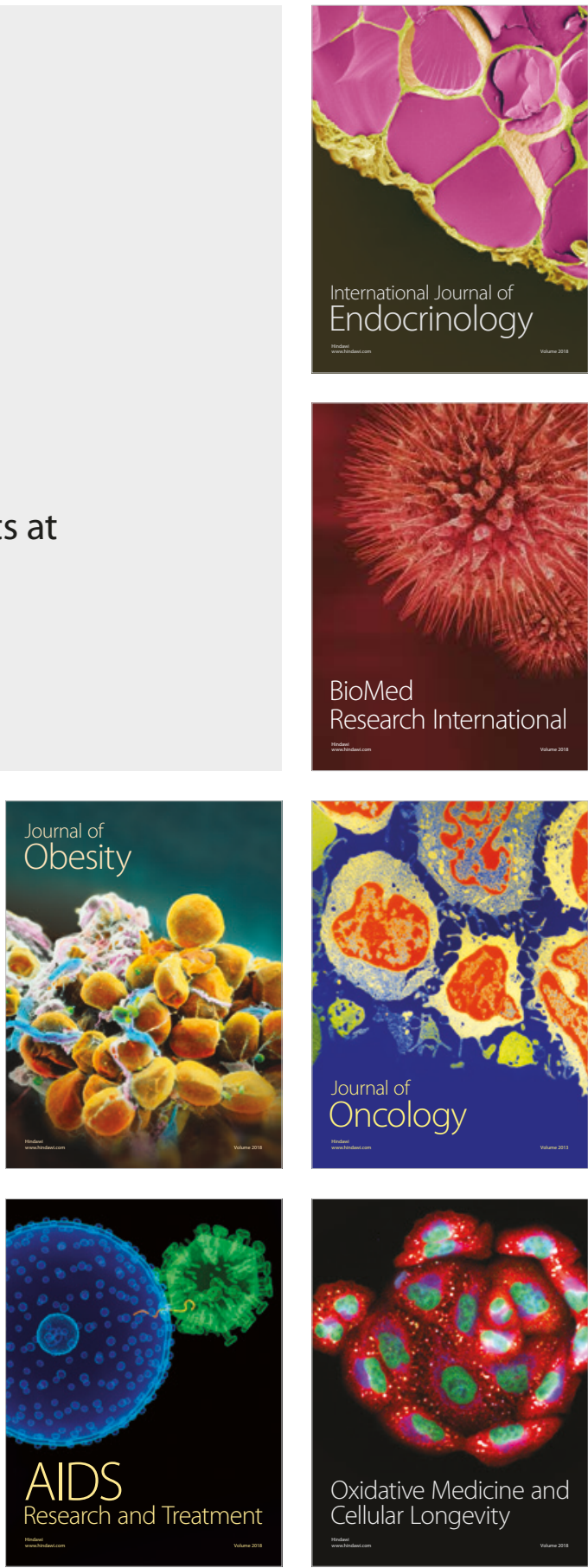